\title{
Adsorption Forms of NO on Iridium-doped Rhodium Clusters in the Gas Phase Revealed by Infrared Multiple Photon Dissociation Spec- troscopy
}

Yufei Zhang ${ }^{\dagger}$, Masato Yamaguchi ${ }^{\dagger}$, Kimihiro Kawada ${ }^{\dagger}$, Satoshi Kudoh ${ }^{\dagger}$, Olga V. Lushchikova ${ }^{\ddagger}$ Joost M. Bakker ${ }^{\ddagger}$ and Fumitaka Mafuné*

$\dagger$ Department of Basic Science, Graduate School of Arts and Sciences, The University of Tokyo, komaba, Meguro-ku, Tokyo 153-8902 (Japan)

*Radboud University, Institute for Molecules and Materials, FELIX laboratory, Toernooiveld 7, 6525 ED Nijmegen (Netherlands)
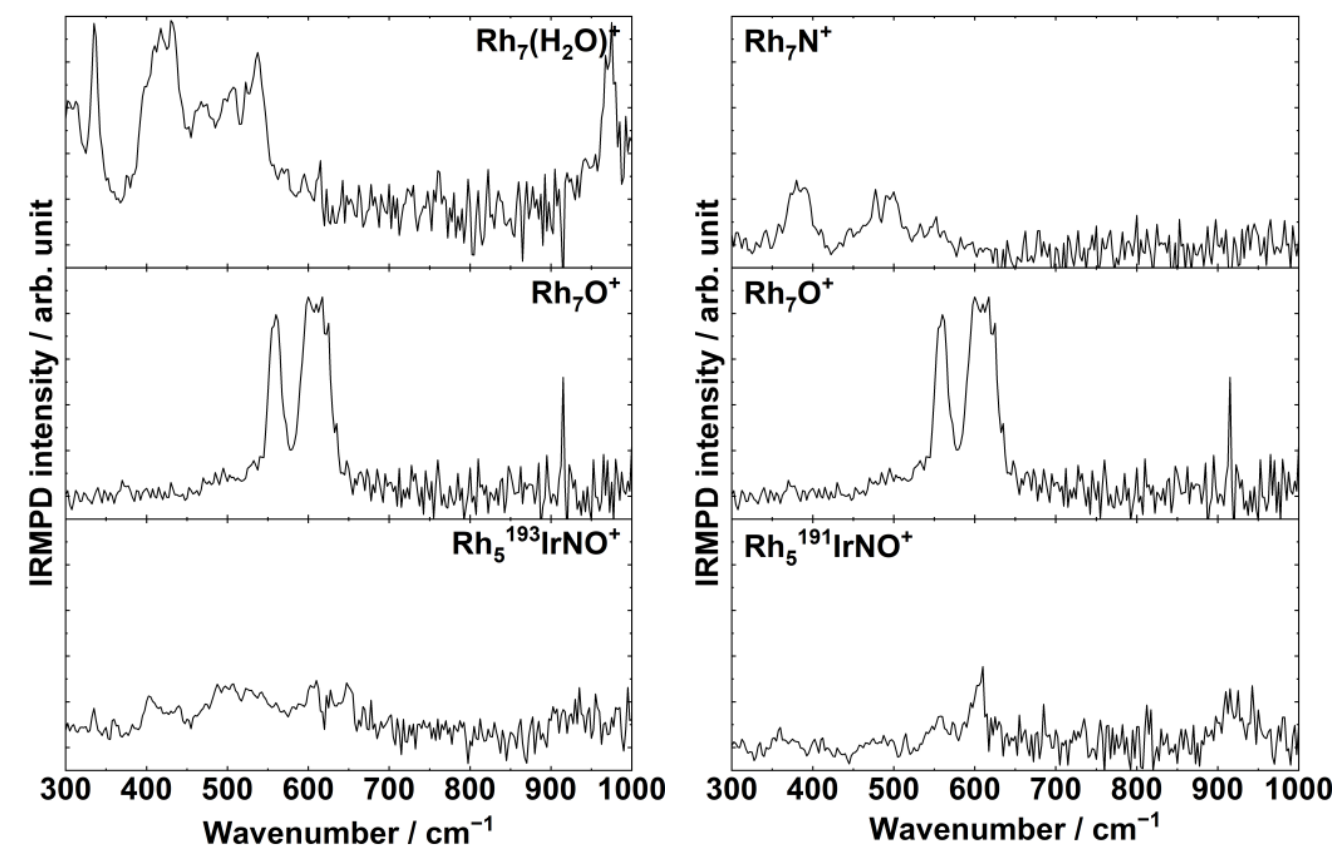

Figure S1 Left: IRMPD spectra with potential interfering mass channels for $\mathrm{Rh}_{5}{ }^{193} \mathrm{IrNO}^{+}$ (m/z= 737.5): $\mathrm{Rh}_{7}\left(\mathrm{H}_{2} \mathrm{O}\right)^{+}$(738.4), and $\mathrm{Rh}_{7} \mathrm{O}^{+}$(737.5). Right: IRMPD spectra with potential interfering mass channels for $\mathrm{Rh}_{5}{ }^{191} \mathrm{IrNO}^{+}(735.5): \mathrm{Rh}_{7} \mathrm{~N}^{+}(734.3)$, and $\mathrm{Rh}_{7} \mathrm{O}^{+}$(737.5). 


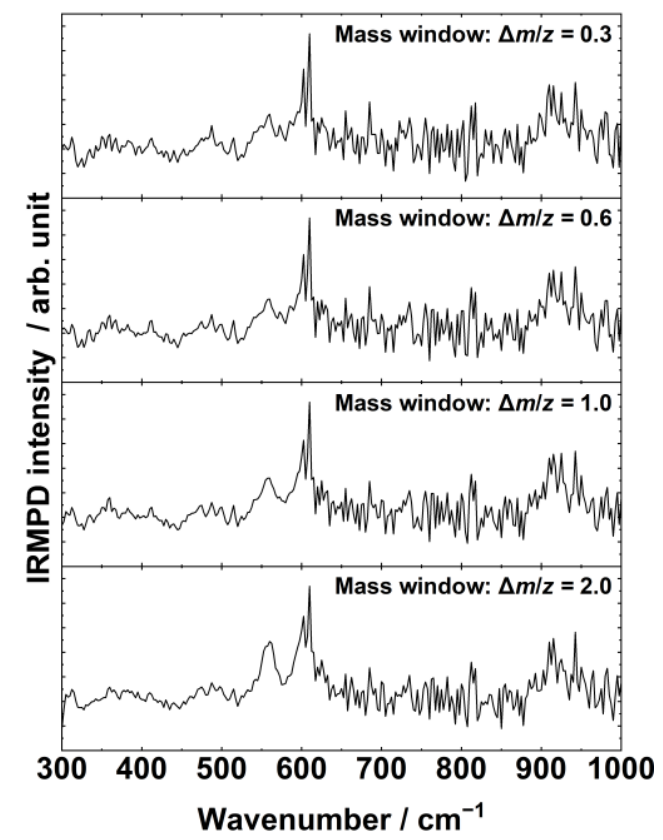

Figure S2 IRMPD spectra of $\mathrm{Rh}_{5}{ }^{191} \mathrm{IrNO}^{+}$for different widths of the mass window.

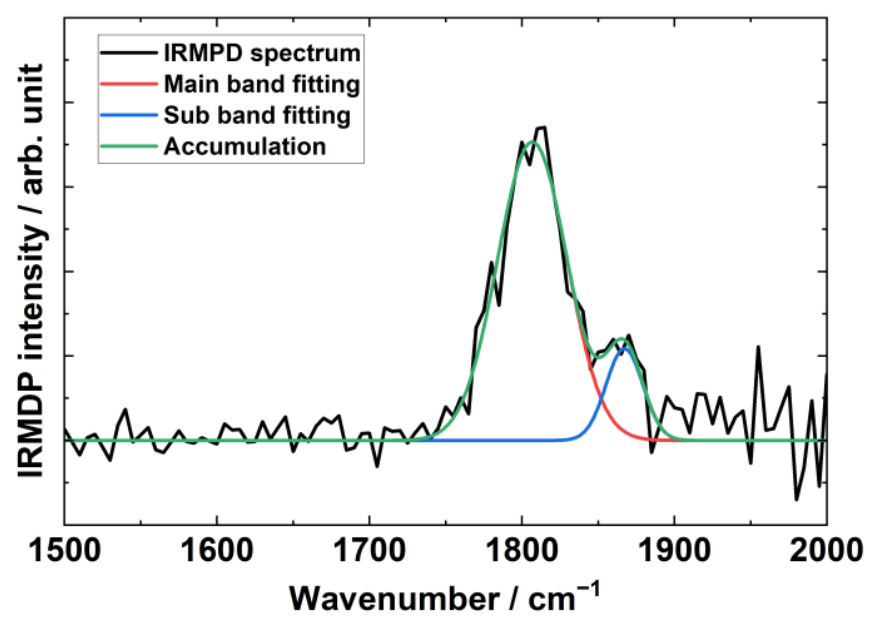

Figure S3 Spectral deconvolution of the $1800 \mathrm{~cm}^{-1}$ band in the IRMPD spectrum of $\mathrm{Rh}_{5} \mathrm{IrNO}^{+}$using two Gaussian lineshape functions, yielding central frequencies of 1806 and $1867 \mathrm{~cm}^{-1}$. 


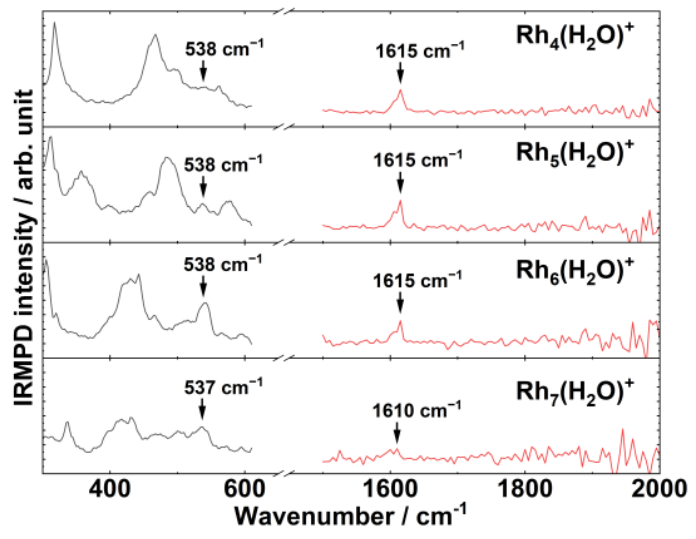

Figure S4 IRMPD spectra of $\mathrm{Rh}_{n}\left(\mathrm{H}_{2} \mathrm{O}\right)^{+}(n=4-7)$.

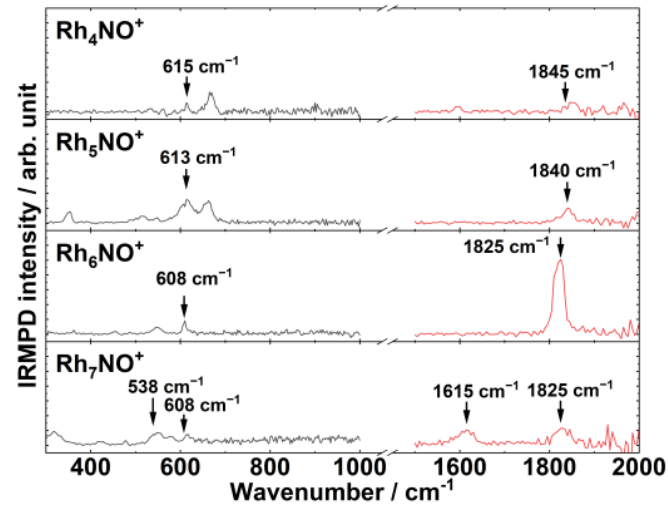

Figure S5 IRMPD spectra of $\mathrm{Rh}_{n} \mathrm{NO}^{+}(n=4-7)$. 


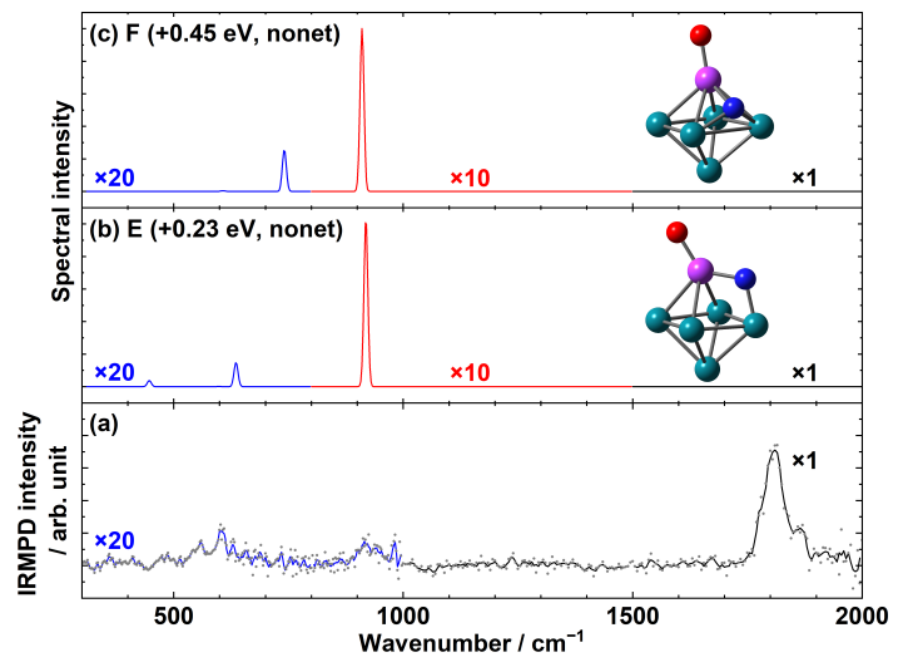

Figure S6 Comparison of IRMPD spectrum and calculated vibrational spectra of second (isomer E) and third (isomer F) stable geometries with dissociative NO . No scaling factor was applied for the wavenumber of calculated vibrational spectra.
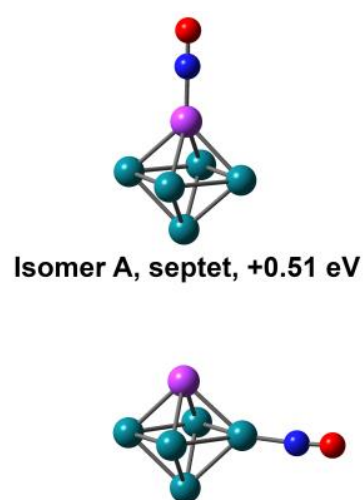

Isomer C, septet, $+1.05 \mathrm{eV}$
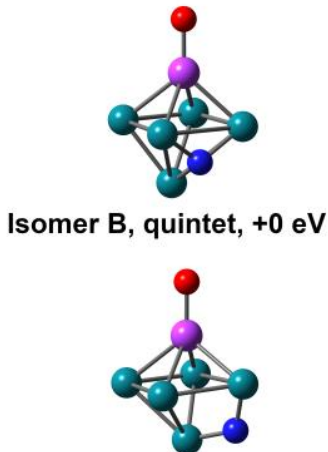

Isomer D, quintet, $+\mathbf{0} .87 \mathrm{eV}$

Figure $\mathbf{S 7}$ Geometrical structures and relative energies of isomer A, B, C and D obtained by calculation applying PBE functional. 

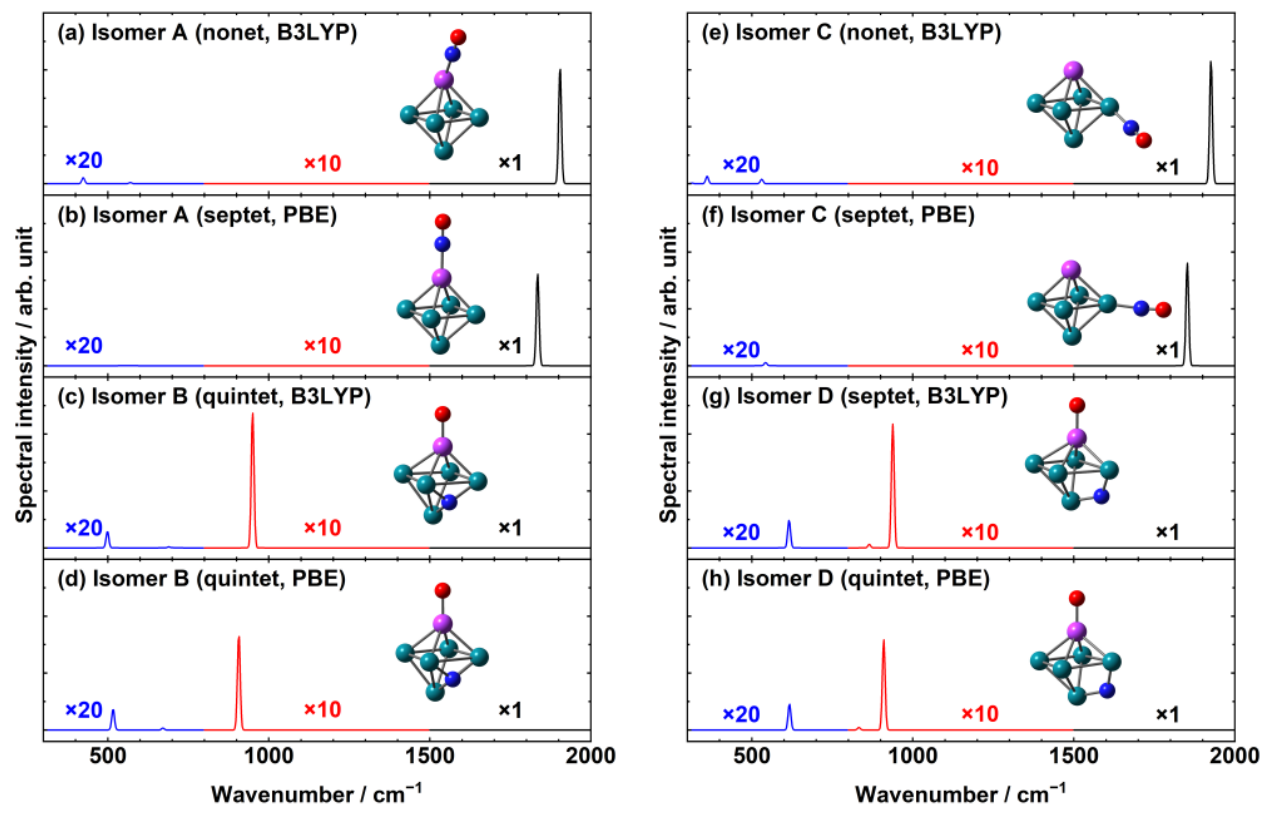

Figure S8 Geometries obtained by PBE functional and their vibrational information.

Table S1 Relative energies (in eV) for the lowest energy isomers compared for the B3LYP and PBE functionals.

\begin{tabular}{|l|l|r|r|r|}
\hline isomer & spin multiplicity & \multicolumn{1}{|l|}{ B3LYP } & spin multiplicity & $P B E$ \\
\hline A & nonet & 0 & septet & 0.51 \\
\hline B & quintet & 0.01 & quintet & 0 \\
\hline C & nonet & 0.51 & septet & 1.05 \\
\hline D & septet & 0.72 & quintet & 0.87 \\
\hline
\end{tabular}

